\author{
1 Universidad de Buenos Aires, Facultad de Agronomía, Cátedra de Botánica General. Buenos Aires, \\ Argentina. *E-mail: torretta@agro.uba.ar \\ ${ }^{2}$ Consejo Nacional de Investigaciones Científicas y Técnicas. Argentina. \\ ${ }^{3}$ Centro de Recursos Naturales Renovables de las Zonas Semiáridas, CONICET. Bahía Blanca, \\ Argentina. \\ ${ }^{4}$ Laboratorio de Interacciones Bióticas en Agroecosistemas (LIBA) - Universidad Nacional del Sur, \\ Departamento de Agronomía. Bahía Blanca, Argentina.
}

Received 08 - XII - 2020| Accepted 23 - IV - 2021| Published 30 - VI - 2021

https://doi.org/10.25085/rsea.800205

\title{
Flower flies (Diptera: Syrphidae) in Pampean agroecosystems: a study case
}

ABSTRACT. Agroecosystems produce profound alterations on the structure and functioning of the original environment, mainly due to the reduction of biodiversity. Flower flies (Diptera: Syrphidae) are potentially useful organisms for the diverse ecosystem services they provide. We studied syrphids collected in a Pampean agroecosystem to assess richness, abundance, host plants, and the ecosystem services they could offer. Flower flies were represented by three subfamilies, eight tribes, 17 genera, and 26 species/morphospecies. Adults of these flies visited flowers of 44 species of wild and/or cultivated plants and exhibited a generalist interaction pattern. Larvae of the most abundant species were predators (mainly soft-bodied Hemiptera) and/or decomposers/aquatic filters. Only one species was pollinivorous. Our results, together with the scarce published bibliography, suggest that the syrphid communities of the highly modified Pampean region are composed of a few very abundant species and many rare or low representative species.

KEYWORDS. Beneficial insects. Biodiversity. Conservation. Ecosystem services. Semi-natural habitats.

RESUMEN. Los agroecosistemas producen profundas alteraciones sobre la estructura y el funcionamiento del ambiente original, debido principalmente a la reducción de la biodiversidad. Las moscas de las flores (Diptera: Syrphidae) son organismos potencialmente útiles por los diversos servicios ecosistémicos que brindan. Se estudiaron los sírfidos colectados en un agroecosistema pampeano para evaluar riqueza, abundancia, plantas huéspedes y los servicios ecosistémicos que podrían ofrecer. Las moscas de las flores estuvieron representadas por tres subfamilias, ocho tribus, 17 géneros y 26 especies/ morfoespecies. Los adultos de estas moscas visitaron flores de 44 especies de plantas silvestres y/o cultivadas y exhibieron un patrón de interacción generalista. Las larvas de las especies más abundantes fueron depredadores (principalmente de hemípteros de cuerpo blando) y/o descomponedores/filtradores. Una única especie fue polinívora. Nuestros resultados, junto a la escasa bibliografía publicada, sugieren que las comunidades de sírfidos de la altamente modificada región pampeana están compuestas por pocas especies muy abundantes y muchas especies raras o con baja representatividad.

PALABRAS CLAVE. Biodiversidad. Conservación. Hábitats semi-naturales. Insectos benéficos. Servicios ecosistémicos. 


\section{INTRODUCCIÓN}

Los agroecosistemas son ambientes naturales modificados con el fin de obtener diversos productos para el aprovechamiento humano (Lombardo et al. 2014). Las modificaciones producidas ejercen profundas alteraciones sobre la estructura y el funcionamiento original, debido principalmente a la reducción de la biodiversidad, generando ecosistemas simplificados y homogéneos sostenidos por el aporte continuo de insumos (trabajo agrícola, agroquímicos y energía fósil) que reemplazan las funciones naturales (Swift et al., 2004). Los agroecosistemas presentan dos componentes de biodiversidad fundamentales: la biodiversidad planificada, que incluye la variedad de especies vegetales y/o animales domesticadas que son incorporados en el ambiente; y la biodiversidad asociada, que incluye organismos cuya presencia no es mediada directamente por acción humana (Altieri, 1999). Generalmente, la biodiversidad asociada es escasamente conocida, aunque cumple importantes roles ecológicos para el sostenimiento de la productividad del agroecosistema. Estos roles son denominados servicios ecosistémicos, entre los que se encuentran la descomposición, el control biológico, la polinización, entre muchos otros (Altieri, 1999).

Los insectos cumplen con numerosos servicios ecosistémicos, y entre ellos, las moscas se destacan por presentar una enorme diversidad morfológica y funcional (McAlpine, 1981, 1987). En particular, las moscas de las flores o sírfidos (Diptera: Syrphidae), conforman una familia cosmopolita de unas 6.200 especies descritas (Young et al., 2016), muy diversa tanto en morfología como en sus ciclos de vida (Vockeroth \& Thompson, 1987). La mayoría de los adultos son polinizadores (Doyle et al., 2020) y los estadios larvales exhiben una gran diversidad de comportamiento alimenticio. Existen especies saprófagas, depredadoras, polínivoras, minadoras de hojas, fitófagas y algunas que pueden producir miasis en humanos y en animales de cría (Thompson et al., 2010; Zuijen \& Nishida, 2011; Pérez-Bañón et al., 2020).

En Argentina existen alrededor de 170 especies de moscas de las flores (López- García et al., 2019), de las cuales cuatro son exóticas (Torretta et al., 2021). De los pocos trabajos realizados sobre esta familia, la mayoría se basa en estudios taxonómicos (detallados en López-García et al., 2019) o en su rol como especies afidófagas (Greco, 1995; López-García \& Maza, 2013; Maza, 2018); sin embargo, aún son escasas las investigaciones sobre esta familia en agroecosistemas y en ambientes naturales. Algunos trabajos acerca de visitantes florales de cultivos y flora acompañante en agroecosistemas de Argentina (principalmente en la región pampeana) citan especies de sírfidos, pero no como el grupo central del estudio (Torretta et al., 2010; Torretta \& Poggio, 2013; Marrero et al., 2014; 2015; 2017; Chamer et al., 2020; Monasterolo et al., 2020). El conocimiento de la fauna nativa presente en agroecosistemas es un factor clave en la gestión y mantenimiento de los mismos (Sommaggio, 1999) y resulta particularmente relevante el estudio en los agroecosistemas de la región Pampeana ya que se desconocen los efectos de la intensa transformación que sufrió esta región en las últimas décadas sobre la fauna silvestre local (Medan et al., 2011).

Los objetivos del presente trabajo fueron 1) conocer la diversidad de sírfidos en un agroecosistema pampeano, 2) reconocer las especies florales utilizadas por los adultos, y 3) indagar sobre el posible rol ecológico de las especies presentes, en relación al comportamiento alimenticio de sus larvas y las visitas florales de los adultos. Finalmente, discutir los resultados obtenidos con la información publicada para otras comunidades de moscas de las flores en agroecosistemas y áreas naturales de la región pampeana.

\section{MATERIAL Y MÉTODOS}

\section{Sitio de estudio}

La Estancia San Claudio (a partir de ahora, SC; partido de Carlos Casares, provincia de Buenos Aires; $\left.36^{\circ} 00^{\prime} \mathrm{S}, 61^{\circ} 50^{\prime} \mathrm{O}\right)$ se encuentra dentro de la subregión Pampa Interior (Marrero et al., 2014). Esta subregión presenta un clima subhúmedo con un promedio de precipitación anual de $1.022 \mathrm{~mm}$ y una temperatura media anual de $15,8{ }^{\circ} \mathrm{C}$ (Tognetti et al., 2010). El área de estudio posee suelos Hapludols típico franco-arenosos, dominantes en la región (Facelli \& D'Angela, 1990). Es un establecimiento agrícolaganadero que posee una superficie aproximada de 5.600 hectáreas, dedicadas en su mayoría a agricultura de manejo tradicional (Marrero et al., 2014). Se utilizan principalmente sistemas de cultivo mixto con aplicación frecuente de agroquímicos (Graffigna et al., 2021). Los principales cultivos de primavera-verano son la soja (Glycine max (L.) Merr.) y el maíz (Zea mays L.), en menor medida el girasol (Helianthus annuus L.) y la alfalfa (Medicago sativa L.). Además existen sectores con árboles y/o pasturas implantadas (Tognetti et al., 2010). Aproximadamente, el $4 \%$ de SC presenta hábitats semi-naturales (por ejemplo, márgenes de cultivos, caminos y áreas peri-domésticas, clausuras) 
TORRETTA, J.P. et al. Sírfidos en agroecosistemas pampeanos

con diversas especies de plantas entomófilas nativas y exóticas (Marrero et al., 2014).

\section{Moscas de las flores: material analizado determinación}

Se analizaron los ejemplares de sírfidos depositados en la Colección Entomológica de la Cátedra de Botánica General de la Facultad de Agronomía (U.B.A.), colectados por J.P. Torretta, y H.J. Marrero (y colaboradores), en SC entre los años 2004 y 2018 (desde noviembre hasta abril, no todos los años fueron muestreados), en distintos trabajos de investigación de ambos autores. Debido a que los ejemplares fueron capturados durante distintos estudios, existen diferencias en la manera que fueron colectados; no obstante, las capturas siempre fueron realizadas con redes entomológicas (Sakagami et al., 1967) en visitas primavero-estivales sobre flores de cultivos y/o especies espontáneas. En algunos casos, se registró la planta huésped mientras que en otros no. Las capturas fueron realizadas en lotes cultivados, márgenes/bordes de los cultivos, en caminos internos de SC y/o en caminos rurales, y en áreas cerradas al manejo agrícola (clausuras). Los insectos capturados fueron sacrificados in situ, en frascos con cianuro de potasio (KCN) y posteriormente fueron montados en alfileres entomológicos y etiquetados (fecha de captura, planta huésped [si tuviera] y colector/es).

Para la determinación de las moscas de las flores se utilizaron las claves de Hull (1946), Lagrange (1987; 1990a; 1990b; 1992), Thompson (1997, 1999; 2006), Thompson et al. (1990, 2000), Thompson \& Thompson (2006), Borges \& Couri (2009) y se actualizó la clasificación taxonómica de algunos géneros (Mengual et al., 2018a, 2018b; Miranda et al., 2020). En otros casos se compararon los especímenes con material de referencia determinado por F.C. Thompson depositados en la propia colección entomológica de la cátedra o en el Museo Argentino de Ciencias Naturales Bernardino Rivadavia (MACN). A los individuos que no lograron ser determinados a nivel específico se los consideró como morfoespecies.

\section{Análisis de los datos}

A partir de los datos obtenidos se calcularon la riqueza (número de especies o morfoespecies) y la abundancia (número de individuos colectados de cada especie o morfoespecie). Se clasificó a cada especie según su origen (nativa o exótica). Para analizar los recursos florales utilizados por las moscas de las flores, se listaron todas las especies de plantas huéspedes y se clasificaron por afinidad taxonómica (familia) y origen (nativa o exótica). Además, se graficó una red de interacción entre las moscas de las flores y sus plantas huéspedes utilizando el paquete bipartite (Dormann et al., 2009) del software estadístico $R$ ( $R$ Development Core Team, 2013); para ello, todas las interacciones observadas durante todos los años de muestreo fueron agrupadas en una única matriz.

Para aquellas especies determinadas fehacientemente, se realizó una búsqueda bibliográfica para registrar el comportamiento alimenticio de las larvas y poder asociarlas a un determinado grupo funcional: descomponedor, filtrador, depredador, de importancia sanitaria (por ejemplo, generador de miasis). Cabe destacar que no se han colectado/ estudiado estadios larvales, sino que los comportamientos alimenticios para las especies/ morfoespecies están inferidos a partir de datos conocidos para las mismas u otra/s especie/s de los géneros.

\section{RESULTADOS}

\section{Diversidad y abundancia}

En total, se analizaron 1.827 individuos adultos capturados a lo largo de todos los años de estudio. Las moscas de las flores de SC estuvieron representadas por tres subfamilias, ocho tribus, 17 géneros y 26 especies/morfoespecies (Tabla I). Todos los géneros capturados estuvieron representados por una o dos especies, excepto Palpada Macquart que estuvo representado por siete especies. De las 26 especies capturadas, 23 son especies nativas y tres son exóticas. Entre las especies nativas, se incluyeron a las cinco morfoespecies, ya que pertenecen a géneros exclusivamente americanos y con especies presentes en el país.

En relación a la riqueza, la subfamilia Eristalinae estuvo representada por ocho géneros y 14 especies (Tabla I), y fue ligeramente superior a la subfamilia Syrphinae (ocho géneros y 11 especies). En cuanto a la abundancia, la relación se invierte: la subfamilia Syrphinae fue la más abundante con 1.106 individuos, mientras que Eristalinae estuvo representada por 720 individuos capturados. La subfamilia Microdontinae solo estuvo representada por un único ejemplar. Sólo tres géneros (Allograpta Osten Sacken, Palpada y Toxomerus Macquart) representaron casi el 91\% de los ejemplares, y solamente dos especies Allograpta exotica Wiedemann (584 ejemplares) y Toxomerus duplicatus (Wiedemann) (483 ejemplares) dominaron la abundancia con el $58,4 \%$ de todas las moscas de las flores colectadas en SC. Veinte especies estuvieron representadas por menos de 25 ejemplares, 15 por seis o menos ejemplares, y cinco por un único ejemplar ("singletons").

\section{Recursos florales utilizados}

Se registraron 576 interacciones entre 22 especies de sírfidos y flores de 44 especies de plantas cultivadas y/o espontáneas (Tabla II) pertenecientes a 15 familias vegetales. Las moscas de las flores forrajearon por recursos alimenticios (néctar y/o polen) en 16 especies nativas y 28 exóticas (o naturalizadas) (Flora Argentina, 2020; Fig. 1) 


\begin{tabular}{|c|c|c|c|c|c|}
\hline Subfamilia & Tribu & Género & Especie & Ejemplares & Origen \\
\hline \multirow{14}{*}{ Eristalinae } & Brachyopini & Lepidomyia & L. ortalina & 3 & $\mathrm{~N}$ \\
\hline & Ceriodini & Sphiximorpha & S. barbipes & 6 & $\mathrm{~N}$ \\
\hline & \multirow{10}{*}{ Eristalini } & Eristalinus & E.taeniops & 4 & E \\
\hline & & Eristalis & E. tenax & 20 & $\mathrm{E}$ \\
\hline & & Meromacrus & M. nectarinoides & 2 & $\mathrm{~N}$ \\
\hline & & \multirow{7}{*}{ Palpada } & P. albiventris & 5 & $\mathrm{~N}$ \\
\hline & & & P. distinguenda & 219 & $\mathrm{~N}$ \\
\hline & & & P.elegans & 217 & $\mathrm{~N}$ \\
\hline & & & P. furcata & 6 & $\mathrm{~N}$ \\
\hline & & & P. meigenii & 24 & $\mathrm{~N}$ \\
\hline & & & P. pusilla & 13 & $\mathrm{~N}$ \\
\hline & & & P. rufiventris & 99 & $\mathrm{~N}$ \\
\hline & Milesiini & Syritta & S. flaviventris & 100 & $\mathrm{E}$ \\
\hline & Volucellini & Copestylum & Copestylum sp. & 2 & $\mathrm{~N}$ \\
\hline Microdontinae & Microdontini & Microdon & Microdon sp. & 1 & $\mathrm{~N}$ \\
\hline \multirow{11}{*}{ Syrphinae } & \multirow{3}{*}{ Bacchini } & Leucopodella & Leucopodella sp. & 1 & $\mathrm{~N}$ \\
\hline & & \multirow{2}{*}{ Platycheirus } & P. (Carposcalis) punctulata & 4 & $\mathrm{~N}$ \\
\hline & & & P. (Carposcalis) saltana & 3 & $\mathrm{~N}$ \\
\hline & \multirow{6}{*}{ Syrphini } & \multirow{2}{*}{ Allograpta } & A. exotica & 584 & $\mathrm{~N}$ \\
\hline & & & A. cf. obliqua & 1 & $\mathrm{~N}$ \\
\hline & & Dioprosopa & D. clavata & 13 & $\mathrm{~N}$ \\
\hline & & Nuntianus & Nuntianus sp. 1 & 1 & $\mathrm{~N}$ \\
\hline & & Syrphus & S. reedi & 5 & $\mathrm{~N}$ \\
\hline & & Victoriana & Victoriana sp. 1 & 1 & $\mathrm{~N}$ \\
\hline & \multirow{2}{*}{ Toxomerini } & \multirow{2}{*}{ Toxomerus } & T. duplicatus & 483 & $\mathrm{~N}$ \\
\hline & & & T.politus & 10 & $\mathrm{~N}$ \\
\hline
\end{tabular}

Tabla I. Diversidad de moscas de las flores (Diptera: Syrphidae) capturadas en flores silvestres y cultivadas en la Estancia San Claudio (C. Casares, Prov. de Buenos Aires), un agroecosistema pampeano. Origen E: exótica; N: nativa.

En relación a la abundancia y riqueza de sírfidos, las flores o inflorescencias más visitadas fueron las de las especies exóticas Hirschfeldia incana (L.) Lagr.-Fossat (38\% de las visitas y $56 \%$ de las especies de sírfidos), Conium maculatum L. ( $17,2 \%$ y $48 \%$, respectivamente), Carduus acanthoides L. (6,4\% y $36 \%$, respectivamente) y la especie nativa Baccharis pingraea DC. (11,3\% y $44 \%$, respectivamente). En total, estas cuatro especies vegetales soportaron al $72,9 \%$ de los individuos colectados y el $72 \%$ de las especies de moscas de las flores presentes en el sitio de estudio.

Servicios ecosistémicos brindados por los estadios larvales

De acuerdo a los ciclos de vida de las moscas de las flores colectadas en SC, a las especies se las puede clasificar en dos grupos funcionales (filtradores/ descomponedores, depredadores) asociados a la 


\begin{tabular}{|c|c|c|c|c|c|c|}
\hline \multirow{2}{*}{ \# } & \multirow{2}{*}{ Familia } & \multirow{2}{*}{ Especie } & \multirow{2}{*}{ Status } & \multicolumn{3}{|c|}{ Adultos colectados } \\
\hline & & & & Abundancia & Géneros & Especies \\
\hline 1 & \multirow{9}{*}{ Apiaceae } & Ammi majus & $\mathrm{E}$ & 2 & 2 & 2 \\
\hline 2 & & Ammi visnaga & E & 9 & 2 & 3 \\
\hline 3 & & Conium maculatum & $\mathrm{E}$ & 99 & 8 & 12 \\
\hline 4 & & Eryngium cabrerae & $\mathrm{N}$ & 1 & 1 & 1 \\
\hline 5 & & Eryngium elegans & $\mathrm{N}$ & 8 & 2 & 4 \\
\hline 6 & & Eryngium horridum & $\mathrm{N}$ & 12 & 4 & 6 \\
\hline 7 & & Eryngium pandanifolium & $\mathrm{N}$ & 6 & 1 & 3 \\
\hline 8 & & Foeniculum vulgare & $\mathrm{E}$ & 3 & 1 & 1 \\
\hline 9 & & Hydrocotyle bonariensis & $\mathrm{N}$ & 1 & 1 & 1 \\
\hline 10 & \multirow{11}{*}{ Asteraceae } & Anthemis cotula & $\mathrm{E}$ & 5 & 2 & 2 \\
\hline 11 & & Baccharis pingraea & $\mathrm{N}$ & 65 & 5 & 11 \\
\hline 12 & & Carduus acanthoides & $\mathrm{E}$ & 37 & 5 & 9 \\
\hline 13 & & Cirsium vulgare & $\mathrm{E}$ & 7 & 3 & 4 \\
\hline 14 & & Crepis setosa & $\mathrm{E}$ & 6 & 2 & 2 \\
\hline 15 & & Eclipta prostrata & $\mathrm{N}$ & 2 & 1 & 2 \\
\hline 16 & & Galinsoga parviflora & $\mathrm{N}$ & 2 & 1 & 1 \\
\hline 17 & & Helianthus annuus & $\mathrm{E}$ & 3 & 1 & 2 \\
\hline 18 & & Hypochoeris pampasica & $\mathrm{N}$ & 8 & 1 & 1 \\
\hline 19 & & Sonchus oleraceus & $\mathrm{E}$ & 9 & 3 & 4 \\
\hline 20 & & Taraxacum officinale & $\mathrm{E}$ & 4 & 2 & 3 \\
\hline 21 & \multirow{4}{*}{ Brassicaceae } & Brassica rapa & $\mathrm{E}$ & 1 & 1 & 1 \\
\hline 22 & & Diplotaxis tenuifolia & $\mathrm{E}$ & 9 & 3 & 4 \\
\hline 23 & & Hirschfeldia incana & $\mathrm{E}$ & 219 & 9 & 14 \\
\hline 24 & & Raphanus sativus & $\mathrm{E}$ & 2 & 1 & 1 \\
\hline 25 & \multirow{4}{*}{ Fabaceae } & Medicago lupulina & $\mathrm{E}$ & 1 & 1 & 1 \\
\hline 26 & & Medicago sativa & $\mathrm{E}$ & 2 & 2 & 2 \\
\hline 27 & & Melilotus albus & $\mathrm{E}$ & 3 & 2 & 3 \\
\hline 28 & & Melilotus officinalis & $\mathrm{E}$ & 3 & 2 & 3 \\
\hline 29 & \multirow{2}{*}{ Geraniaceae } & Erodium $\mathrm{sp}$. & $E$ & 3 & 1 & 1 \\
\hline 30 & & Geranium sp. & $\mathrm{N}$ & 4 & 1 & 1 \\
\hline 31 & Lamiaceae & Marrubium vulgare & E & 1 & 1 & 1 \\
\hline 32 & Myrtaceae & Eucalyptus sp. & $\mathrm{E}$ & 3 & 1 & 1 \\
\hline 33 & Oleaceae & Ligustrum lucidum & $\mathrm{E}$ & 2 & 2 & 2 \\
\hline 34 & Oxalidaceae & Oxalis sp. & $\mathrm{N}$ & 1 & 1 & 1 \\
\hline 35 & \multirow{2}{*}{ Plantaginaceae } & Plantago sp. & $E$ & 1 & 1 & 1 \\
\hline 36 & & Verbascum thapsus & E & 1 & 1 & 1 \\
\hline 37 & Polygonacaee & Polygonum persicaria & $\mathrm{E}$ & 9 & 3 & 4 \\
\hline 38 & Portulacaceae & Portulaca oleracea & $\mathrm{E}$ & 5 & 2 & 4 \\
\hline 39 & Solanaceae & Solanum sisymbriifolium & $\mathrm{N}$ & 6 & 3 & 3 \\
\hline 40 & Turneraceae & Turnera sidioides & $\mathrm{N}$ & 1 & 1 & 1 \\
\hline 41 & \multirow{4}{*}{ Verbenaceae } & Glandularia peruviana & $\mathrm{N}$ & 1 & 1 & 1 \\
\hline 42 & & Verbena bonariensis & $\mathrm{N}$ & 4 & 3 & 4 \\
\hline 43 & & Verbena gracilescens & $\mathrm{N}$ & 10 & 4 & 6 \\
\hline 44 & & Verbena intermedia & $\mathrm{N}$ & 2 & 2 & 2 \\
\hline
\end{tabular}

Tabla II. Plantas huéspedes de las moscas de las flores (Diptera: Syrphidae) capturadas en la Estancia San Claudio (C. Casares, Prov. de Buenos Aires), un agroecosistema pampeano. Origen: $E$ = exótica; $N$ = nativa. 
subfamilia a la que pertenecen (Tabla III). El 39,4\% de los individuos (subfamilia Eristalinae) capturados se pueden catalogar como filtradores de ítems orgánicos en aguas polutas y/o descomponedores. De las 14 especies/morfoespecies de Eristalinae capturadas hay datos bibliográficos para cinco de ellas (35,7\%); de las cuales tres son sinantrópicas y exóticas (Tabla III).

Por otro lado, el resto de los individuos (60\%) se los puede catalogar como depredadores (subfamilias Syrphinae [excepto Toxomerus politus (Say)] y Microdontinae). La mayoría de las especies de Syrphinae son predadores de insectos de cuerpo blando (aunque existen especies polinívoras, minadoras de hojas). De las 11 especies/morfoespecies de Syrphinae capturadas hay datos bibliográficos para siete de ellas (Tabla III). La mayoría de las especies con ciclo de vida conocido resultaron ser depredadoras de áfidos, y para $T$. duplicatus además hay registros de consumo de larvas de dos especies de escarabajos del género Microtheca Stål (Coleoptera: Chrysomelidae) en el sur de Brasil. La excepción dentro de la subfamilia Syrphinae es T. politus cuyas larvas se alimentan sobre granos de polen de maíz (Z. mays [Poaceae]; Tabla III), un cultivo estival abundante en el área de estudio. Por lo tanto, los diez individuos capturados de esta especie $(0,6 \%)$ deben ser catalogados como polinívoros y no estarían brindado un servicio ecosistémico). Finalmente, al único individuo capturado de Microdontinae se lo puede clasificar también como depredador, debido al comportamiento de sus larvas de alimentarse de estadios inmaduros de hormigas (López-García et al., 2020).

\section{DISCUSIÓN}

\section{Diversidad}

Este trabajo representa el primer análisis de la comunidad de moscas de las flores en un agroecosistema o en un ambiente natural de Argentina. Los resultados muestran que la comunidad de moscas de las flores en el agroecosistema estudiado estuvo dominada por un pequeño grupo de especies muy abundantes y muchas especies poco frecuentes. Estas últimas pueden representar más del $50 \%$ de las especies colectadas en comunidades de insectos tropicales, incluso en grandes colecciones (Novotny \& Basset, 2000).

Debido a los diferentes requerimientos de hábitats, los sírfidos podrían verse particularmente afectados por una disminución en la diversidad de paisajes (Sommaggio, 1999), tal como ocurre en hábitats homogéneos (es decir, grandes áreas de monocultivo) y profundamente modificados como los agroecosistemas pampeanos. La dominancia numérica de A. exotica y T. duplicatus podría deberse a sus comportamientos

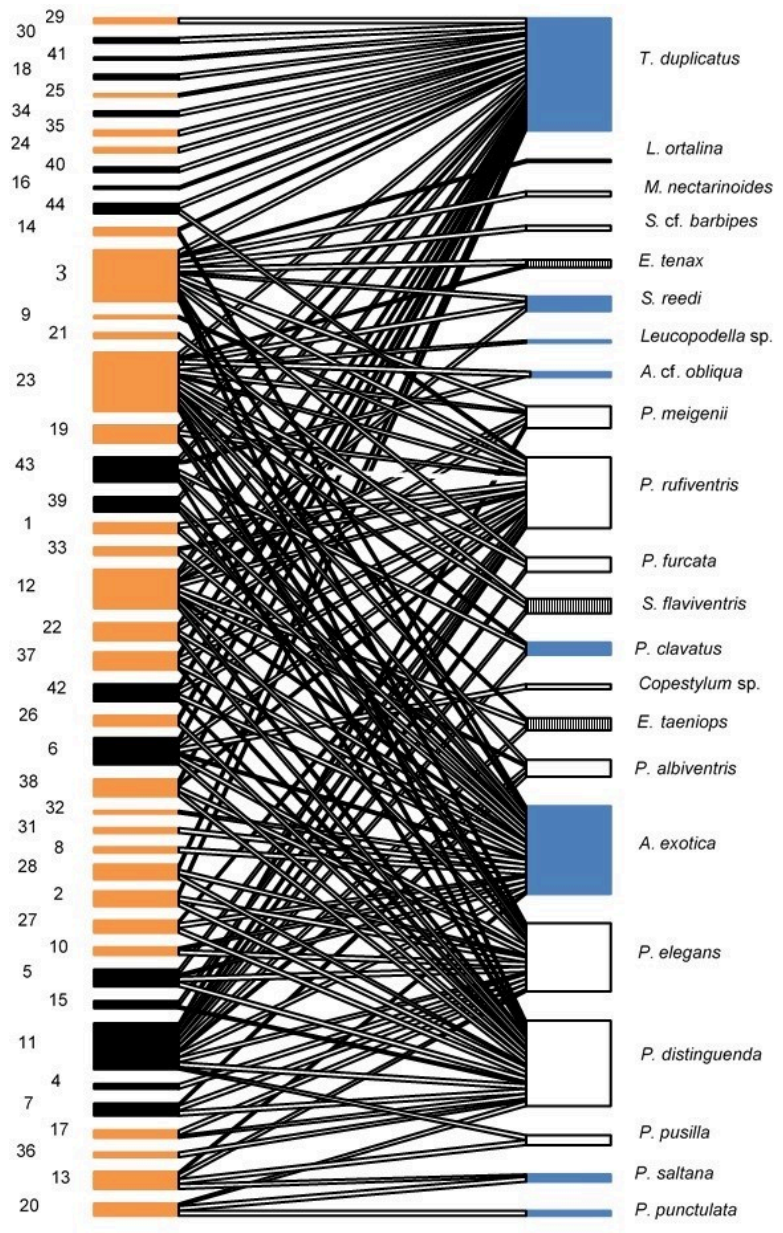

Fig. 1. Red cuantitativa de interacciones entre las moscas de las flores (Diptera: Syrphidae) y sus plantas huéspedes en la Estancia San Claudio (C. Casares, Prov. de Buenos Aires), un agroecosistema pampeano. Las especies están representadas por rectángulos cuyo ancho es proporcional a la suma de interacciones que las involucran. Las especies de plantas (el número se corresponde con el de la Tabla II) están discriminadas por origen (nativas: negro; exóticas: naranja) y las especies de moscas de las flores a nivel de Subfamilia (Eristalinae: blanco; Syrphinae: azul). Los rectángulos rayados indican a las especies exóticas de Syrphidae. El ancho de las líneas que representan las interacciones entre las flores y las especies de sírfidos es proporcional a la cantidad de las mismas.

de forrajeo dentro de la matriz agrícola, es decir, que los lotes cultivados sean hábitats favorables para estas especies. Resultados similares fueron reportados para agroecosistemas en el sur de Brasil, donde las tres especies más abundantes representaron entre 81\% (Medeiros et al., 2018) y el 85,5\% (Bortolotto et al., 2016) de los individuos colectados, y $A$. exotica fue la especie 
más abundante en estos trabajos, representando el 52\% (Medeiros et al., 2018) y el 60,5\% (Bortolotto et al., 2016). Allograpta exotica es una especie muy abundante en su rango de distribución natural (Arcaya et al., 2017), con adultos activos durante el invierno en la región pampeana y probablemente exhiba un ciclo de vida mutivoltino (varias generaciones al año) (Greco, 1995). Souza et al. (2014) reportan que las comunidades de sírfidos en ambientes abiertos y de temprana sucesión ecológica, tal como los agroecosistemas, están dominadas por pocas especies muy abundantes; mientras que son más equitativas (con menor riqueza y similar abundancia entre especies) en bosques bien conservados. Es llamativa la baja abundancia de $T$. politus, una de las pocas especies fitófagas del género (Reemer \& Rotheray, 2009), cuyas larvas se desarrollan en granos de polen de maíz, un recurso muy abundante (al menos temporalmente) en el sitio de estudio. Sin embrago, debido a que los muestreos estuvieron sesgados hacia flores de plantas entomófilas, la representatividad real de esta especie podría ser mayor que la reportada en este trabajo.

El género Palpada estuvo representado por siete de las 27 reportadas en el país (Lagrange, 1992); la presencia de casi $26 \%$ de la riqueza nacional es llamativo para un agroecosistema altamente modificado como SC. Nuevamente, la mayor abundancia de individuos (ca. 92\%) correspondió solamente a tres especies, con muy pocos ejemplares de las restantes. El suelo de la Pampa Interior, en la cual está situado nuestro sitio de estudio, presenta una topografía extremadamente chata, con un lento escurrimiento (Aragón et al., 2011). Este tipo de suelo, permite la formación de cuerpos de agua temporales donde las hembras adultas de estas especies podrían encontrar sitios aptos para la oviposición.

La baja representatividad de la subfamilia Microdontinae podría deberse a que los adultos no forrajean en flores; por lo tanto, el tipo de muestreo realizado (captura con redes entomológicas sobre flores) podría estar sesgando su presencia. Otra posible explicación podría ser que el manejo del agroecosistema (utilización de herbicidas y pesticidas, y otras prácticas agrícolas) disminuya la disponibilidad de nidos de hormigas.

\section{Recursos florales utilizados}

Las moscas de las flores forrajearon por néctar y/o polen en numerosas especies de plantas silvestres $\mathrm{y} /$ o espontáneas presentes en los márgenes lineales de los lotes cultivados, en los caminos rurales y/o en áreas semi-naturales dentro del agroecosistema estudiado.

El número de plantas entomófilas presentes en SC y sus alrededores exhibe variabilidad interanual y geográfica: Marrero et al. (2014) citan 71 especies (bordes de cultivos, clausuras), Rosanigo et al. (2020) informan 48 especies (solo en las clausuras) y Monasterolo et al. (2020) reportan 72 especies en los caminos rurales. Las moscas de las flores utilizaron 44 especies vegetales con diferentes morfologías florales (formas, colores, tamaños, etc.). Las especies vegetales espontáneas más abundantes, y con mayores fenologías de floración $(H$. incana, $C$. acanthoides, $B$. pingraea; obs. pers.) fueron la más utilizadas por los sírfidos. Otra especie vegetal exótica muy utilizada fue C. maculatum (Apiaceae), en la cual se han capturado muchas de las especies raras (es decir, poco colectadas): Lepidomyia ortalina Wulp, Meromacrus nectarinoides Lynch Arrabálzaga y Sphiximorpha barbipes Loew, solo capturadas en sus flores. Esta especie vegetal es una de las primeras en florecer, con el pico de abundancia floral en noviembre-diciembre, con una fenología de floración explosiva y con gran cantidad de recursos (néctar y/o polen) para sus polinizadores, como ocurre con otras Apiaceae (Lenardis et al., 2017). Posiblemente, en esa época del año sea una de las principales fuentes de recurso para los polinizadores. A excepción de B. pingraea, que forma manchones perennes, en general asociado a la base grandes árboles implantados (ej. Eucaliptus sp.; obs. pers.), las plantas nativas tienden a ser escasas y su oferta floral limitada. Por lo tanto, la comunidad de moscas de las flores en SC (y posiblemente en casi todos los agroecosistemas pampeanos) depende de los recursos aportados por la planta silvestres exóticas para mantener sus poblaciones. Esto mismo sucede con muchas otras especies de polinizadores presentes en nuestro sitio de estudio (Torretta \& Durante, 2011; Torretta et al., 2012; Marrero et al., 2014).

Nuestros resultados muestran que los sírfidos de SC son visitantes florales generalistas como ya ha sido reportado generalmente para la familia (Lucas et al., 2018). La red de interacciones muestra que las visitas de los sírfidos a las flores/inflorescencias exhibe un patrón anidado, es decir, que hay un grupo de especies centrales generalistas que visitan plantas muy frecuentadas y otro grupo de plantas poco visitadas por otros sírfidos, y muchas especies especialistas (o raras) que visitan a plantas muy visitadas; un típico patrón observado en redes mutualistas planta-polinizador (Bascompte et al., 2003). Klecka et al. (2020) muestran que existe mayor especialización en especies de Eristalinae y Pipizinae comparadas con especies más generalistas de Syrphinae, sin embargo, nuestros datos están fuertemente dominados por muy pocas especies, por lo que cualquier conclusión parece especulativa.

\section{Servicios ecosistémicos}

Las larvas de las especies colectadas en nuestro sitio de estudio ofrecen dos importantes servicios ecosistémicos: control biológico y descomposición. Las especies de la subfamilia Syrphinae se caracterizan por ser depredadoras voraces de áfidos y pulgones, alimentándose de numerosos individuos a lo largo de su desarrollo (Rojo et al., 2003; Arcaya et al., 2017). Este hábito alimentario las convierte en potenciales 


\begin{tabular}{|c|c|c|c|}
\hline Subfamilia & Especie & Grupo & Cita \\
\hline \multirow{7}{*}{ Eristalinae } & \multirow{2}{*}{ Eristalinus taeniops } & Descomponedor/filtrador & Pérez-Bañón et al., 2003 \\
\hline & & Productor de miasis & Pérez-Bañón et al., 2020 \\
\hline & \multirow{2}{*}{ Eristalis tenax } & Descomponedor/filtrador & Thompson, 1997 \\
\hline & & Productor de miasis & Kun et al., 1998 \\
\hline & Palpada furcata & Descomponedor en restos de café & Pérez-Bañón et al., 2003 \\
\hline & Sphiximorpha barbipes & Descomponedor en huecos de árboles y tallos de bambú & Ricarte et al., 2007 \\
\hline & Syritta flaviventris & Descomponedor (en cladodios de Opuntia) & Pérez-Bañón \& Marcos-García, 2000 \\
\hline \multirow{8}{*}{ Syrphinae } & Allograpta exotica & Depredador (afidófago) & Greco, 1995; Arcaya et al., 2017; Maza, 2018 \\
\hline & Allograpta obliqua & Depredador (afidófago) & Greco, 1995; Maza, 2018 \\
\hline & Dioprosopa clavata & Depredador (afidófago) & Greco, 1995; López-García \& Maza, 2013 \\
\hline & Platycheirus (Carposcalis) punctulata & Depredador (afidófago) & López-García \& Maza, 2013 \\
\hline & Platycheirus (Carposcalis) saltana & Depredador (afidófago) & López-García \& Maza, 2013 \\
\hline & \multirow{2}{*}{ Toxomerus duplicatus } & Depredador (afidófago) & Maza, 2018 \\
\hline & & Depredador sobre larva de crisomélido & Sturza et al., 2014 \\
\hline & Toxomerus politus & Consumidor de granos de polen de maíz (fitófago) & Reemer \& Rotheray, 2009 \\
\hline
\end{tabular}

Tabla III. Datos biológicos bibliográficos para especies de moscas de las flores (Diptera: Syrphidae) capturadas en la Estancia San Claudio (C. Casares, Prov. de Buenos Aires), un agroecosistema pampeano.

controladores biológicos dentro de los ecosistemas (Tenhumberg \& Poehling, 1995; Maza, 2018). Las larvas de la subfamilia Eristalinae son saprófagas en materia en descomposición de diversos orígenes y/o en aguas polutas (Mengual et al., 2008). Existe escasa información sobre los hábitos particulares de saprofagia de las especies estudiadas en SC. Las especies de Palpada, debido a su abundancia, se presentan como grupos particularmente interesantes para ser estudiados en cuanto su aporte en el ciclado de nutrientes en el agroecosistema.

Por otro lado, los adultos de dos de las tres subfamilias presentes en SC utilizan polen y/o néctar de flores para alimentarse. Debido a su abundancia y a su capacidad de transportar polen a través de largas distancias, las moscas de las flores podrían ser consideradas polinizadoras generalistas (Vockeroth \& Thompson, 1987; Pérez-Bañón et al., 2007) y de gran importancia para las especies vegetales silvestres de SC. Algunos autores (Rader et al., 2016; Doyle et al., 2020) mencionan a los sírfidos como los dípteros polinizadores más importantes y más frecuentes después de los himenópteros. Sin embargo, su eficiencia en la polinización continúa en estudio. Según Holloway (1976), los miembros de la subfamilia Eristalinae podrían ser más eficientes respecto a los de la subfamilia Syrphinae, debido a la menor densidad de pilosidad y los frecuentes hábitos de acicalamiento de éstos últimos.

Finalmente, debido a su capacidad potencial para producir miasis, se deberían controlar las poblaciones de Eristalis tenax (L.) (Kun et al., 1998) y Eristalinus taeniops Wiedemann (Pérez-Bañón et al., 2020).

Comparación con otros ensambles de sírfidos en agroecosistemas y sistemas naturales pampeanos

La información acerca de la comunidad de moscas de las flores en agroecosistemas pampeanos es escasa. Greco (1995) estudió la fenología de las seis especies afidófagas más comunes en parcelas de cultivos agrícolas; de las cuales tres están presentes en SC: A. exotica, A. obliqua (Say) y Dioprosopa clavata (Fabricius) (citada como Pseudodoros clavatus (Fabricius)) y las otras tres especies pertenecen a tres géneros también presentes en SC (Platycheirus Lepeletier \& Serville, Ocyptamus s.l. Macquart y Syrphus Fabricius). Otros trabajos en los cuales se menciona la presencia de syrphidae en agroecosistemas pampeanos, se focalizaron en interacciones de malezas, plantas silvestres (de bordes de cultivos, áreas semi-naturales, etc.) y/o cultivos y sus potenciales polinizadores. En lotes cultivados de girasol y la vegetación asociada en cercanías de Balcarce (3746’ S, 58¹8' O), Torretta \& Poggio (2013) reportan solamente la presencia de A. exotica y Palpada elegans Blanchard. Marrero et al. (2014) presentan datos de visitantes florales colectados en lotes ganaderos cercanos a la localidad Pila (36 $16^{\prime} \mathrm{S}, 5^{\circ} 1^{\prime}$ O) durante las estaciones primavero-estivales de tres años consecutivos (2009-2011) y reportan siete especies de sírfidos, de las cuales seis están presentes en SC: $A$. exotica, T. duplicatus (citado como Toxomerus sp.) y cuatro especies de Palpada ( $P$. distinguenda Wiedemann, $P$. elegans, $P$. meigenii Wiedemann y $P$. rufiventris (Macquart)). Para lotes de girasol y la vegetación asociada, en Gral. Villegas (3443' S, 6312 $\mathrm{O})$, en el NO de la provincia de Buenos Aires, Chamer et al. (2020) mencionan a Allograpta sp., Palpada sp. y Toxomerus sp. Por último, Monasterolo et al. (2020) presentan datos de visitantes florales de flores silvestres en caminos rurales que rodean a SC (el mismo sitio de estudio que este trabajo); y reportan 14 especies de sírfidos: Palpada (6 spp.; sin mencionar abundancias específicas), Toxomerus (2 spp.) y Allograpta (1 sp.) representan las especies más abundantes.

Asimismo, también existen pocos registros de 
moscas de las flores en sitios naturales y/o protegidos de la región pampeana. Nuevamente, en base de trabajos relacionados a la interacción de plantaspolinizadores, se puede encontrar alguna información. En los bosques de tala protegidos en la Reserva de la Biosfera MAB-UNESCO 'Parque Costero del Sur' (35 $\mathrm{S}$, 57³0' O), Basilio et al. (2006) capturaron 13 especies de sírfidos durante tres años (desde agosto 1998 hasta mayo 2001). El elenco de sírfidos de estos bosques estuvo dominado nuevamente por Palpada (6 spp.), A. exotica, T. duplicatus (citado como Toxomerus sp.), con la presencia en mucha menor abundancia de individuos de D. clavata, Syrphus reedi Shannon, Platycheirus (Carposcalis) spp., Copestylum sp., entre otros. Estos autores no listan las especies, pero los ejemplares pudieron ser estudiados debido a que se encuentran depositados en la Colección Entomológica de la Cátedra de Botánica General (FAUBA). En la Reserva

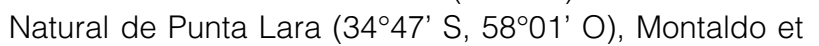
al. (2017) reportan 10 especies de sírfidos en flores de seis especies de plantas leñosas, pero no indican sus abundancias; con la presencia de Allograpta, Palpada, Toxomerus, y Ocyptamus s.I. Por otro lado, en el parque provincial Ernesto Tornquist (3803' S, 6202' O), Haedo et al. (2017) evaluaron la atracción de las flores de plantas nativas silvestres hacia los visitantes florales con el fin de promover su utilización en agroecosistemas para mejorar la conservación de los polinizadores. Los autores reportan 11 especies de moscas de las flores pertenecientes a los géneros Palpada (4 spp.), Copestylum Macquart (3 spp.), y a las especies $A$. exotica (como Allograpta sp.), T. duplicatus (como Toxomerus sp.), E. tenax (como Eristalis sp.) y Orthonevra argentina (Brèthes) (como especie indeterminada). Finalmente, Sabatino et al. (2010) estudiaron redes de interacción planta-polinizador en 12 sierras del sistema Tandilia entre las localidades de Mar

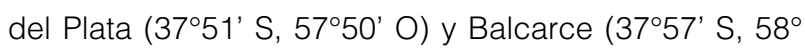
$\left.49^{\prime} \mathrm{O}\right)$. En las sierras están presentes especies de moscas de las flores pertenecientes a los géneros Allograpta, Palpada, Toxomerus (en todas las sierras muestreadas), Platycheirus (nueve sierras), Copestylum (cuatro sierras), Dioprosopa (como Pseudodoros, una sierra) y tres especies exóticas Syritta flaviventris Macquart (como Syritta sp., tres sierras), E. taeniops (dos sierras) y E. tenax (una sierra), y otras dos especies no determinadas observadas en una sierra cada una (Gilarranz et al., 2015). Por lo tanto, el número de especies de moscas de las flores en la región pampeana parece ser baja y estar dominado por algunas pocas especies muy abundantes. En todos los sitios con datos, la presencia de $A$. exotica, $T$. duplicatus y algunas especies de Palpada (principalmente $P$. distinguenda y $P$. elegans) es prácticamente constante. Por otro lado, hay especies de algunos géneros que se encuentran presentes en varios de los sitios relevados, por ejemplo: $D$. clavata, Platycheirus (Carposcalis) spp., S. reedi, otras especies de Palpada (como P. rufiventris, P. meigenii, y P. furcata Wiedemman), Copestylum sp., aunque en abundancias bajas. La presencia de especies poco colectadas en estos ambientes pampeanos, por ejemplo L. ortalina, $M$. nectarinoides, S. barbipes, Leucopodella sp., Nuntianus $\mathrm{sp}$. y Victoriana sp. (estas dos últimas anteriormente en Ocyptamus s.l.) podría ser más errática y depender de las condiciones ambientales o del estado de conservación del ecosistema. La presencia de especies exóticas aumenta la diversidad y la abundancia de estas moscas en la región pampeana, sin embargo, hasta el momento no hay trabajos que evalúen competencia entre especies exóticas y nativas.

El conocimiento de la diversidad de insectos es considerado esencial para establecer la calidad de los servicios ecosistémicos en los ambientes (Garibaldi et al., 2020). En particular para las moscas de las flores, es escasa la información sobre su biodiversidad en los agroecosistemas de nuestro país, incluso conociendo la potencialidad de ofrecer diversos servicios ecosistémicos (Dunn et al., 2020). Por lo tanto, son necesarios estudios que integren diferentes diversos aspectos de la ecología de esta importante familia de moscas, y la promoción de estudios sistemáticos que ayuden a mejorar la resolución taxonómica de muchas de las especies del país.

\section{AGRADECIMIENTOS}

Agradecemos a la Universidad de Buenos Aires, por el apoyo logístico y el permiso para conducir nuestras investigaciones en la Ea. San Claudio. Y al personal de la Estancia por su cordialidad. A M. Rosanigo, S. Olea, J. Sciarra, G. Cilla, G.A.R. Molina y P.R. Mulieri por su colaboración en el trabajo de campo. A X. Mengual por su colaboración en la determinación de los individuos de Nuntianus y Victoriana. A L. Patitucci, M. Sabatino y dos revisores anónimos por los comentarios constructivos del manuscrito. El trabajo de campo fue financiado por la Agencia Nacional de Promoción Científica y Tecnológica: PICT 2006-00851, PICT 08-12504, PICT 2015-2523, PICT 2017-3336 y la Universidad de Buenos Aires G065. Este trabajo formó parte de la Tesis de Licenciatura de M.C.L.

\section{BIBLIOGRAFÍA CITADA}

Altieri, M.A. (1999) The ecological role of biodiversity in agroecosystems. Agriculture, Ecosystems \& Environment, 74, 19-31.

Aragón, R., Jobbagy, E.G., \& Viglizzo, E.F. (2011) Surface and groundwater dynamics in the sedimentary plains of the Western Pampas (Argentina). Ecohydrology, 4, 433-447.

Arcaya, E.A., Pérez-Bañón, C., Mengual, X., Zubcoff-Vallejo, J.J., \& Rojo, S. (2017) Life table and predation rates of the syrphid fly Allograpta exotica, a control agent of the cowpea aphid Aphis craccivora. Biological Control, 115, 74-84. 
Revista de la Sociedad Entomológica Argentina 80(2): 23-34, 2021

Bascompte, J., Jordano, P., Melián, C., \& Olesen, J.M. (2003) The nested assembly of plant-animal mutualistic networks. Proceedings of the National Academy of Sciences, 100 9383-9387.

Basilio, A.M., Medan, D., Torretta, J.P., \& Bartoloni, N. (2006) A year-long plant-pollinator network. Austral Ecology, 31, 975-983.

Borges, Z.M., \& Couri, M.S. (2009) Revision of Toxomerus Macquart, 1855 (Diptera: Syrphidae) from Brazil with synonymic notes, identification key to the species and description of three new species. Zootaxa, 2179, 1-72.

Bortolotto, O.C., Oliveira, A.M.J., Hoshino, A.T., \& Campos, T.A (2016) Distance from the edge of forest fragments influence the abundance of aphidophagous hoverflies (Diptera: Syrphidae) in wheat fields. Acta Scientiarum, 38(2), 157-164.

Chamer, A.M., Medan, D., Montaldo, N.H., Mantese, A.I., \& Devoto, M. (2020) Visitantes florales del girasol (Helianthus annuus) y de su vegetación acompañante en la Pampa Interior. Ecología Austral, 30, 228-238.

Dormann, C.F., Fründ, J., Blüthgen, N., \& Gruber, B. (2009) Indices, graphs and null models: analyzing bipartite ecological networks. Open Ecology Journal, 2(1), 7-24.

Doyle, T., Hawkes, W.L.S., Massy, R., Powney, G.D., Menz, M.H.M., \& Wotton, K.R. (2020) Pollination by hoverflies in the Anthropocene. Proceedings of the Royal Society London (B), 287, 20200508.

Dunn, L., Lequerica, M., Reid, C.R., \& Latty, T. (2020) Dual ecosystem services of syrphid flies (Diptera: Syrphidae): pollinators and biological control agents. Pest Management Science, 76(6), 1973-1979.

Facelli, J.M., \& D'Angela, E. (1990) Directionality, convergence, and rate of change during early succession in the Inland Pampa, Argentina. Journal of Vegetation Science, 1(2), 255-260.

Flora Argentina (2020) http://www.floraargentina.edu.ar/ [Acceso: 10-08-2020].

Garibaldi, L.A., Sáez, A., Aizen, M.A., Fijen, T., \& Bartomeus, I. (2020) Crop pollination management needs flower-visitor monitoring and target values. Journal of Applied Ecology, 57(4), 664-670.

Gilarranz, L.J., Sabatino, M., Aizen, M.A., \& Bascompte, J. (2015) Data From: Hot spots of mutualistic networks. Digital Repository, https://doi.org/10.5061/dryad.cr3ft

Graffigna, S., Marrero, H.J., \& Torretta, J.P. (2021) Glyphosate commercial formulation negatively affects the reproductive success of solitary wild bees in a Pampean agroecosystem. Apidologie, 52, 272-281.

Greco, C.F. (1995) Fenología y selección de hábitat de las especies de sírfidos afidófagos (Dipt.: Syrphidae) más frecuentes en cultivos cerealeros y forrajeros en la provincia de Buenos Aires, Argentina. Entomophaga, 40(3-4), 317-320.

Haedo, J.P., Stalldecker, P., \& Marrero, H.J. (2017) Plantas nativas del sudoeste bonaerense potencialmente útiles para la conservación de los polinizadores en agroecosistemas. BioScriba, 8(1), 12-23.

Holloway, B. (1976) Pollen-feeding in hover-flies (Diptera: Syrphidae). New Zealand Journal of Zoology, 3, 339-350.

Hull, F.K. (1946) The genus Lepidostola. American Museum Novitates, 1326, 1-15.
Klecka, J., Hadrava, J., Biella, P., \& Akter, A. (2018) Flower visitation by hoverflies (Diptera: Syrphidae) in a temperate plant-pollinator network. PeerJ, 6, e6025.

Kun, M., Kreiter, A., \& Semenas, L. (1998) Myiasis gastrointestinal humana por Eristalis tenax. Revista de Saúde Pública, 32, 367-369.

Lagrange, E.B. (1987) Notas sobre el género Palpada Macquart, 1834 (Diptera, Syrphidae). Neotrópica, 32, 97-103.

Lagrange, E.B. (1990a) Las especies argentinas del género Meromacrus Rondani (Diptera, Syrphidae). Revista Brasileira de Entomologia, 34, 489-498.

Lagrange, E.B. (1990b) Revisión de las especies argentinas del grupo agrorum del género Palpada Macquart, 1834 (Diptera, Syrphidae). Revista de la Asociación de Ciencias Naturales del Litoral, 21, 3-40.

Lagrange, E.B. (1992) Revisión de las especies argentinas del grupo vinetorum del genero Palpada Macquart, 1834 (Diptera, Syrphidae). Revista de la Sociedad Entomológica Argentina, 50(1-4), 145-166.

Lenardis, A.E., Gil, A., Torretta, J.P., Ganly, D., Bouilly, J.P., \& de la Fuente, E.B. (2017) Floral visitor assemblages related to coriander genotypes and sowing dates: relationships with volatile signals. NJAS-Wageningen Journal of Life, 83, 22-29.

Lombardo, P., Fernández, P., Moya, M., Sainato, C., Borodowski, E., Muschietti Piana, P., Pescio, F., Acosta, A., \& Urricariet, S. (2014) Agroecosistemas. Caracterización, implicancias ambientales y socioeconómicas. Editorial Facultad de Agronomía (UBA), Buenos Aires.

López-García, G., \& Maza, N. (2013) Lista de sírfidos afidófagos y primeros registros de Pseudodoros clavatus y Eupeodes rojasi (Diptera: Syrphidae), potenciales agentes de control biológico en la provincia de Mendoza, Argentina. Revista de la Sociedad Entomológica Argentina, 72, 237-240.

Lopez-García, G., Barahona-Segovia, R.M., Maza, N., Domínguez, M.C., \& Mengual, X. (2019) Filling gaps in flower fly distributions: first record of Aneriophora aureorufa (Philippi, 1865) (Diptera, Syrphidae) from Argentina. Check List, 15, 249-255.

López-García, G., Reemer, M., Debandi, G., \& Mengual, X. (2020) New information about the third stage larva and larval habitat of Microdon (Chymophila) bruchi Shannon, 1927 (Diptera, Syrphidae) from Argentina. Journal of Natural History, 53, 2833-2853.

Lucas, A., Bodger, O., Brosi, B.J., Ford, C.R., Forman, D.W., Greig, C., Hegarty, M., Neyland, P.J., \& De Vere, N. (2018) Generalisation and specialisation in hoverfly (Syrphidae) grassland pollen transport networks revealed by DNA metabarcoding. Journal of Animal Ecology, 87(4), 1008-1021.

Marrero, H.J., Torretta, J.P., \& Medan, D. (2014) Effect of land use intensification on specialization in plant-floral visitor interaction networks in the Pampas of Argentina. Agriculture, Ecosystems \& Environment, 188, 63-71.

Marrero, H.J., Pompozzi, G., \& Torretta, J.P. (2015) Preferencia de presas y sitios de capturas de arañas cangrejo (Araneae: Thomisidae y Philodromidae) en un pastizal del centro de Argentina. Ecología Austral, 25, 19-25.

Marrero, H.J., Torretta, J.P., Vázquez, D.P., Hodara, K., \& Medan, D. (2017) Exotic plants promote pollination niche overlap in an agroecosystem. Agriculture, Ecosystems \& Environment, 239, 304-309. 
Maza, N. (2018) Potencialidad de sírfidos (Diptera: Syrphydae) como agentes de control biológico de plagas en cultivos de pimiento en invernadero. Tesis Doctoral. Facultad de Agronomía y Zootecnia. Universidad Nacional de Tucumán. Argentina.

McAlpine, J.F. (1981) Manual of Nearctic Diptera. Vol. 1. Biosystematics Research Institute, Ottawa, Ontario, Canada

McAlpine, J.F. (1987) Manual of Nearctic Diptera. Vol. 2. Biosystematics Research Institute, Ottawa, Ontario, Canada.

Medan, D., Torretta, J.P., Hodara, K., de la Fuente, E.B., \& Montaldo, N.H. (2011) Effects of agriculture expansion and intensification on the vertebrate and invertebrate diversity in the Pampas of Argentina. Biodiversity and Conservation, 20, 3077-3100

Medeiros, H.R., Hoshino, A.T., Ribeiro, M.C., Morales, N.M., Martello, F., Pereira, O.C.N., Carstense, D.W., \& Oliveira, A.J.M. (2018) Non-crop habitats modulate alpha and beta diversity of flower flies (Diptera, Syrphidae) in Brazilian agricultural landscapes. Biodiversity and Conservation, $27,1309-1326$

Mengual, X., Stahls, G., \& Rojo, S. (2008) First phylogeny of predatory flower flies (Diptera, Syrphidae, Syrphinae) using mitochondrial COI and nuclear 28S rRNA genes: conflict and congruence with the concurrent tribal classification. Cladistics, 24, 543-562.

Mengual, X., Miranda, G.F.G., \& Thompson, F.C. (2018a) Unraveling Ocyptamus and the Baccha legacy (Diptera: Syrphidae): redefinition of groups and new species descriptions. Zootaxa, 4461(1), 1-44.

Mengual, X., Stahl, G., Laska, P., Maznek, L., \& Rojo, S. (2018b) Molecular phylogenetics of the predatory lineage of flower flies Eupeodes.Scaeva (Diptera: Syrphidae), with the description of the Neotropical genus Austroscaeva gen. nov. Journal of Zoological Systematics and Evolutionary Research, 56, 148-169.

Miranda, G.F.G., Skevington, J.H., \& Marshall, S.A. (2020) New generic concepts for orphaned lineages formerly treated as part of the genus Ocyptamus Macquart, 1834 (Diptera Syrphidae). Zootaxa, 4822(2), 151-174

Monasterolo, M., Poggio, S.L., Medan, D., \& Devoto, M. (2020) Wider road verges sustain higher plant species richness and pollinator abundance in intensively managed agroecosystems. Agriculture Ecosystems \& Environment, 302,107084

Montaldo, N.H., Mantese, A.I., \& Roitman, G.G. (2017) Sistema reproductivo y polinización de especies leñosas en una selva subtropical invadida por plantas exóticas. Boletín de la Sociedad Argentina de Botánica, 52(4), 675-687.

Novotny, V., \& Basset, Y. (2000) Rare species in communities of tropical insect herbivores: pondering the mystery of singletons. Oikos, 89, 564-572.

Pérez-Bañón, C., \& Marcos-García, M.A. (2000) Description of the immature stages of Syritta flaviventris (Diptera: Syrphidae) and new data about the life history of European species of Syritta on Opuntia maxima. European Journal of Entomology, 97(1), 131-136.

Pérez-Bañón, C., Rotheray, G., Hancock, G., Marcos-García, M.A., \& Zumbado, M.A. (2003) Immature stages and breeding sites of some Neotropical saprophagous syrphids (Diptera: Syrphidae). Annals of the Entomological Society of America, 96(4), 468-471.
Pérez-Bañón, C., Petanidou, T., \& Marcos-García, M.A. (2007) Pollination in small islands by occasional visitors: the case of Daucus carota subsp. commutatus (Apiaceae) in the Columbretes archipelago, Spain. Plant Ecology, 192(1), 133-151.

Pérez-Bañón, C., Rojas, C., Vargas, M., Mengual, X., \& Rojo, S. (2020) A world review of reported myiases caused by flower flies (Diptera: Syrphidae), including the first case of human myiasis from Palpada scutellaris (Fabricius, 1805). Parasitology Research, 119, 815-840.

R Development Core Team (2013) R: A language and environment for statistical computing. R Foundation for Statistical Computing, Vienna, Austria. Disponible en: http://www.R-project.org

Rader, R., Bartomeus, I., Garibaldi, L.A., Garratt, M.P., Howlett, B.G., Winfree, R., Cunningham, S.A., Mayfield, M.M., Arthur, A.D., et al. (2016) Non-bee insects are important contributors to global crop pollination. Proceedings of the National Academy of Sciences of the United States of America, 113(1), 1461-1451.

Reemer, N., \& Rotheray, G.E. (2009) Pollen feeding larvae in the presumed predatory syrphine genus Toxomerus Macquart (Diptera, Syrphidae). Journal of Natural History, 43(15-16), 939-949.

Ricarte, A., Marcos-García, M.A., Rotheray, G.E., \& Hancock, E.G. (2007) The early stages and breeding sites of 10 Cerioidini flies (Diptera: Syrphidae). Annals of the Entomological Society of America, 100(6), 914-924.

Rojo, S., Gilbert, F., Marcos-García, M.A., Nieto, J.M., \& Mier, M.P. (2003) A world review of predatory hoverflies (Diptera, Syrphidae: Syrphinae) and their prey. CIBIO, Alicante, España.

Rosanigo, M.P, Marrero, H.J., \& Torretta, J.P. (2020) Limiting resources on the reproductive success of a cavity-nesting bee species in a grassland agroecosystem. Journal of Apicultural Research, 59, 583-591.

Sabatino, M., Maceira, N., \& Aizen, M.A. (2010) Direct effects of habitat area on interaction diversity in pollination webs. Ecological Applications, 20, 1491-1497.

Sakagami, S.F., Laroca, S., \& Moure, J.S. (1967) Wild bee biocenotics in São José dos Pinhais (PR), South Brazil. Preliminary report. Journal of the Faculty of Science, Hokkaido University, Zoology, 16, 253-291.

Sommaggio, D. (1999) Syrphidae: can they be used as environmental bioindicators? Agriculture, Ecosystems \& Environment, 74, 343-356.

Souza, J.M.T., Marinoni, R.C., \& Marinoni, L. (2014) Open and disturbed habitats suport higher diversity of Syrphidae (Diptera)? A case study during three years of sampling in a fragment of araucaria forest in Southern Brazil. Journal of Insect Science, 14, 1-8.

Sturza, V.S., Dequech, S.T.B., Toebe, M., Silveira, T.R., Cargnelutti Filho, A., \& Bolzan, A. (2014) Toxomerus duplicatus Wiedemann, 1830 (Diptera: Syrphidae) preying on Microtheca spp. (Coleoptera: Chrysomelidae) larvae. Brazilian Journal of Biology, 74(3), 656-658.

Swift, M.J., Izac, A.-M.N., \& van Noordwijk, M. (2004) Biodiversity and ecosystem services in agricultural landscapes - are we asking the right questions? Agriculture, Ecosystems \& Environment, 104, 113-134.

Tenhumberg, B., \& Poehling, H. (1995) Syrphids as natural enemies of cereal aphids in Germany: Aspects of their biology and efficacy in different years and regions. Agriculture, Ecosystems and Environment, 52, 39-43. 
Revista de la Sociedad Entomológica Argentina 80(2): 23-34, 2021

Thompson, F.C. (1997) Revision of the Eristalis flower flies (Diptera: Syrphidae) of the Americas South of the United States. Proceedings of the Entomological Society of Washington, 99, 209-237.

Thompson, F.C. (1999) A key to the genera of the flower flies (Diptera: Syrphidae) of the Neotropical region including descriptions of new genera and species and a glossary of taxonomic terms. Contributions on Entomology, International, 3, 321-373.

Thompson, F.C. (2006) Primer taller de identificación de Syrphidae del Neotrópico. Universidad del Valle, Facultad de Ciencias, Cali, Colombia.

Thompson, F.C., \& Thompson, B.J. (2006) A new Toxomerus species from Chile (Diptera: Syrphidae). Studia Dipterologica, 13, 317-331.

Thompson, F.C., Fee, F.D., \& Berzark, L.G. (1990) Two immigrant synanthropic flower flies (Diptera: Syrphidae) new to North America. Entomological News, 101(2), 69-74.

Thompson, F.C., Thompson, B.J., \& Fairman, J.E. (2000) Only in Costa Rica: new Neotropical flower flies (Diptera: Syrphidae). Studia Dipterologica, 7, 33-43.

Thompson, F.C., Rotheray, G.E., \& Zumbado, M.A. (2010) Family Syrphidae. Manual of Central American Diptera. Vol. 2. (ed. Brown, B.V., Borkent, A., Cumming, J.M., Wood, D.M., Woodley, N.E., \& Zumbado M.A.), pp. 763-792. Ottawa: NRC Research Press.

Tognetti, P.M., Chaneton, E.J., Omacini, M., Trebino, H.J., \& León, R.J.C. (2010) Exotic vs. native plant dominance over 20 years of old-field succession on set-aside farmland in Argentina. Biological Conservation, 143, 2494-2503.

Torretta, J.P., \& Durante, S.P. (2011) Nesting ecology of Megachile (Sayapis) mendozana Cockerell and its synonymy with Megachile (Sayapis) santiaguensis Durante (Hymenoptera: Megachilidae). Zootaxa, 3008, 63-68.
Torretta, J.P., \& Poggio, S.L. (2013) Species diversity of entomophilous plants and flower-visiting insects is sustained in the field margins of sunflower crops. Journal of Natural History, 47, 139-165.

Torretta, J.P., Medan, D., Roig-Alsina, A., \& Montaldo, N.H. (2010) Visitantes florales diurnos del girasol (Helianthus annuus, Asterales: Asteraceae) en Argentina. Revista de la Sociedad Entomológica Argentina, 69, 17-32.

Torretta, J.P., Durante, S.P., Colombo, M.G., \& Basilio, A.M. (2012) Nesting biology of the leafcutting bee Megachile (Pseudocentron) gomphrenoides (Hymenoptera: Megachilidae) in an agro-ecosystem. Apidologie, 43, 624-633.

Torretta, J.P., Haedo, J.P., Allasino, M.L., \& Marrero, H.J. (2021) First records of the phytophagous Eumerus strigatus (Fallén) (Diptera: Syrphidae: Syrphinae: Merodontini) in Argentina. Revista de la Sociedad Entomológica Argentina, 80(1), 93-95.

Vockeroth, J.R., \& Thompson, F.C. (1987) Syrphidae. Manual of Nearctic Diptera, vol. 2. (ed. McAlpine, J.F.), pp 713-743. Canada Communication Group Pub., Canada.

Young, A.D., Lemmon, A.R., Skevington, J.H., Mengual, X., Stahls, G., Reemer, M., Jordaens, K., Kelso, S., Lemmon, E.M., et al. (2016) Anchored enrichment dataset for true flies (order Diptera) reveals insights into the phylogeny of flower flies (family Syrphidae). BMC Evolutionary Biology, 16, 143.

Zuijen, M.P., \& Nishida, K. (2011) Description of life history and immature stages of phytophagous flower fly, Allograpta zumbadoi Thompson (Diptera: Syrphidae: Syrphinae). Studia Dipterologica, 17, 37-51. 